\title{
Catéter-fístula: una nueva alternativa en la punción de accesos vasculares
}

\author{
Premio Izasa Accesos Vasculares y Nuevas Tecnologías
}

Olga Martínez Ocaña* - Jaime Rodríguez Estaire*- Beatriz Ruiz Sanz* - Juan Antonio Martín Navarro**_ Eva Mérida Herrero**

*Diplomados en Enfermería, ** Nefrólogos

Centro de Hemodiálisis San Luciano. Fresenius Medical Care. Madrid

\section{Resumen}

Es imprescindible un acceso vascular adecuado para obtener una hemodiálisis óptima. El daño causado por la punción de la aguja convencional o complicaciones intradiálisis derivadas del movimiento del brazo pueden comprometer la supervivencia del acceso. Otro factor esencial en la calidad de la hemodiálisis, íntimamente relacionado con el calibre de la aguja, es conseguir un mayor flujo sanguíneo eficaz.

El catéter-fístula, una nueva cánula de punción de fluoruroplástico, disminuye el daño en la pared interna del acceso y presenta un menor calibre de punción con un mayor calibre interior, lo que le permite alcanzar mayores flujos con una máxima eficacia depuradora y menores complicaciones vasculares.

El objetivo de este estudio prospectivo fue comparar el uso del catéter-fístula con el uso de la aguja convencional. Se realizaron 18 sesiones de hemodiálisis con cada uno de los dispositivos en pacientes estables y con buen acceso vascular, recogiéndose en todos los casos el flujo obtenido, las presiones y otros parámetros del monitor, analíticas e incidencias.

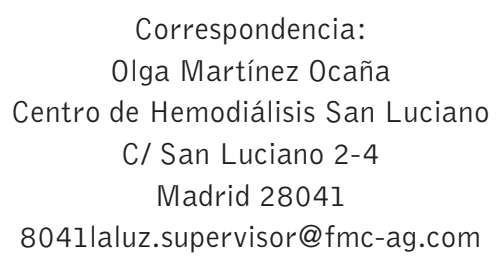

Los resultados han demostrado que la utilización del catéter-fístula disminuyó la presión arterial y la presión venosa, sin disminuir la eficacia dialítica, en consecuencia, mejoró el perfil hemodinámico. Mejoró significativamente el flujo de sangre real, el número de litros dializados y el Kt.

El menor trauma mecánico producido en el acceso vascular permitió disminuir la dosis de heparina, el tiempo de hemostasia y hubo una reducción de las complicaciones vasculares: extravasación y resangrado. Siendo bien tolerada la punción, hay un leve empeoramiento en la sensación dolorosa, justificada por la inexperiencia del manejo.

La mejoría en los parámetros dinámicos abren las posibilidades de conseguir mayor eficacia dialítica al hacer factible la utilización de mayores flujos sanguíneos con presiones más adecuadas.

PALABRAS CLAVE:

- CATETER FISTULA

- ACCESO VASCULAR

- PUNCIÓN

- PRESION VENOSA

- FLUJO SANGRE 


\section{Fistula catheter: a new alternative in venous access puncture}

\section{Abstract}

Adequate vascular access is essential in order to obtain optimal haemodialysis. The damage caused by conventional needle puncture or intradialytic complications deriving from the movement of the arm can compromise the survival of the access. Another essential factor in the quality of the haemodialysis, closely related to the calibre of the needle, is obtaining a greater efficient blood flow.

The fistula catheter, a new puncture cannula made of fluoroplastic, reduces the damage to the internal wall of the access and presents a lower puncture calibre with a higher interior calibre, which allows it to reach better flows with maximum efficiency and fewer vascular complications.

The aim of this prospective study was to compare the use of the fistula catheter with the use of the needle. Eighteen haemodialysis sessions were carried out with each of the devices in stable patients with good vascular access, recording in all cases the flow obtained, pressures and other monitor parameters, test results and incidents.

The results have shown that the use of the fistula catheter reduced the arterial pressure and venous pressure, without reducing the dialytic efficiency, and consequently the haemodynamic profile improved. The actual blood flow, number of litres dialysed and Kt improved significantly.

The lesser mechanical trauma caused in the vascular access made it possible to reduce the dose of heparin, the haemostasis time and there was a reduction in vascular complications: extravasation and rebleeding. While the puncture is well tolerated, there is a slight worsening in the feeling of pain, explained by the lack of experience in handling.

The improvement in dynamic parameters opens up the possibilities of achieving greater dialytic efficiency by making it feasible to use greater blood flows with more adequate pressures.

\section{KEY WORDS: \\ - FISTULA CATHETER \\ - VASCULAR ACCESS \\ - PUNCTURE \\ - VENOUS PRESSURE \\ - BLOOD FLOW}

\section{Introducción}

El acceso vascular (AV) adecuado es un requisito imprescindible para proporcionar una diálisis óptimal. La fístula arteriovenosa interna (FAVI) es el acceso vascular de elección en pacientes en hemodiálisis (HD), aunque no es el único, existiendo otros alternativos, siendo el más empleado el injerto de politetrafluoretileno expandido (PTFE) ${ }^{2}$.

El daño a FAVIs e injertos, causado por la punción de agujas convencionales o por el movimiento o flexión del brazo durante la diálisis, puede ser el principal factor en la longevidad del AV. Otro factor a tener en cuenta relacionado con el aclaramiento de solutos durante la HD es el flujo de sangre y para que éste sea adecuado es importante considerar el calibre de la aguja ${ }^{3}$. Es por todo ello, que consideramos que tanto la punción del AV como la utilización de un calibre de aguja apropiado son factores esenciales en la calidad de HD.

En este sentido, se ha desarrollado recientemente, una nueva cánula de punción, el catéter-fístula, caracterizado por ser un catéter de fluoruoplástico que evita el riesgo de daño en la pared interna del injerto o FAVI en situaciones de movimiento o flexión del brazo, además de presentar con un calibre de punción menor, un mayor calibre en el interior del $\mathrm{AV}$, que permite alcanzar flujos estables y elevar el flujo sanguíneo, logrando así, la máxima eficacia depuradora de toxinas. Todas estas características abren interesantes expectativas relacionadas con su mejor tolerabilidad, adecuación dialítica y disminución de complicaciones.

\section{Objetivos}

\section{Objetivo General:}

Comparar el catéter-fístula con la aguja convencional durante la sesión de diálisis. 


\section{Objetivos Específicos:}

- Comprobar si el catéter-fístula influye en parámetros dinámicos tales como Presión Venosa (PV), Presión Arterial (PA) y Presión Transmembrana (PTM).

- Verificar si el calibre del catéter-fístula influye en el flujo de sangre real y litros dializados así como en la eficacia dialítica.

- Relacionar si el uso del catéter-fístula influye en la dosis de heparina, en los tiempos de hemostasia y complicaciones, tales como resangrado postsesión y extravasaciones.

- Comparar la tolerabilidad y dolor a la punción del catéter-fístula con la aguja convencional.

\section{Material y métodos}

\section{Descripción del catéter- fístula}

El catéter-fístula Supercath $A Z^{\circledR}$, altamente biocompatible, es una aguja metálica interna tribiselada para la punción, revestida de un catéter de fluoruroplástico sin soldaduras, con un acabado muy fino de punta roma, que presenta un orificio central (luz del catéter) y orificios laterales en la punta del catéter (figura 1). El calibre de la aguja interna es $16 \mathrm{G}$ y el diámetro interno del catéter es 14G. Está equipado con una válvula antiretorno, que evita el goteo de sangre y un sistema de revestimiento telescópico de la aguja que elimina los pinchazos accidentales.

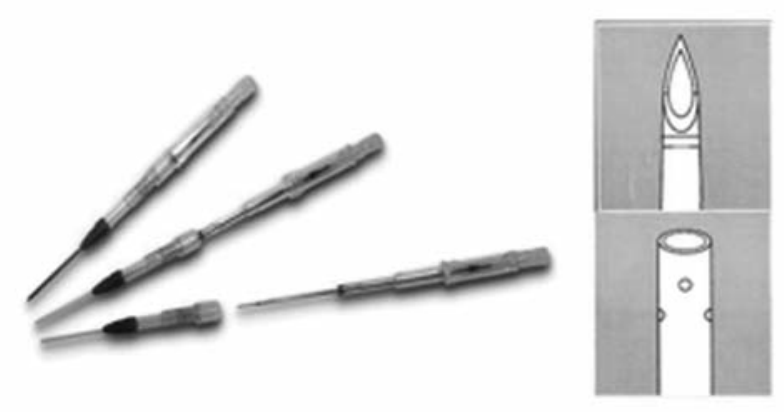

Figura 1. Descripción del catéter fístula Supercath $A Z^{\circledR}$

La técnica de punción requiere sujetar el catéter-fístula entre el conector y la válvula anti-retorno para evitar que en el momento de la punción se retraiga la aguja metálica. Una vez puncionada la vena progresar el catéter en la luz del AV a la vez que se retira la aguja metálica y se fija el catéter-fístula a la piel (figura 2).
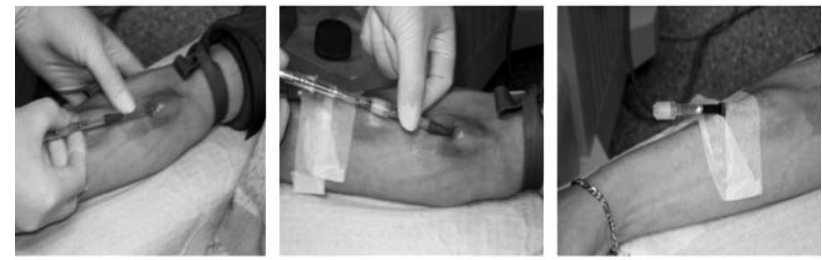

Figura 2. Técnica de punción del Supercath $A Z^{\circledR}$

\section{Método}

Se realizó un estudio prospectivo de 3 meses de duración (diciembre 2008-marzo 2009) en el centro de Hemodiálisis San Luciano, en el cual se modificó el tipo de cánula de punción según el siguiente esquema: 18 sesiones con aguja convencional calibre $15 \mathrm{G}$ y 18 sesiones con catéter-fístula Supercath $A Z^{\circledR}$ calibre 16G. Los pacientes se seleccionaron entre los 132 pacientes de nuestro centro en tratamiento sustitutivo renal mediante HD convencional.

Los criterios de inclusión fueron los siguientes:

1. Portador de FAVI autóloga cuyo acceso arterial o venoso se punciona en la flexura del codo y portador de injerto PTFE, normofuncionantes, durante un período superior a 3 meses.

2. Paciente estable, sin ingresar en los últimos 6 meses.

3. Acceso vascular que proporciona un flujo de bomba de sangre mínimo de $300 \mathrm{ml} / \mathrm{min}$ con una PV $\leq 200$ $\mathrm{mm} \mathrm{Hg}$ y un tiempo mínimo de utilización de 10 meses, sin complicaciones por causa vascular en los últimos 3 meses.

4. Sin antecedentes a alergias a agujas esterilizadas con óxido de etileno.

Se incluyeron a 10 pacientes, 9 hombres y 1 mujer, con una edad media de 69 años (58-88 años). Todos los pacientes estaban en programa de HD convencional, 3 días por semana, con una duración media de 232, 5 min (210- $240 \mathrm{~min}$ ), con membrana de alta permeabilidad, un flujo de bomba de sangre medio de $384 \mathrm{ml} / \mathrm{min}$ (350$400 \mathrm{ml} / \mathrm{min}$ ), en monitor Fresenius $4008 \mathrm{~S}^{\circledR}$. En cuanto al flujo del baño, 8 pacientes tenían flujo de $500 \mathrm{ml} / \mathrm{min}$ y 2 pacientes de $800 \mathrm{ml} / \mathrm{min}$. Así mismo, 1 paciente era portador de injerto PTFE y 9 pacientes de FAVI, de los cuales 3 eran radiocefálicas y 6 húmero-cefálicas. 
Para la valoración se diseñó una hoja de recogida de datos, en la que se registraron valores como PA, PV y PTM iniciales y finales, flujo de bomba en sangre, flujo de sangre real, litros dializados, ultrafiltración pautada, episodios de hipotensión, extravasación y sangrado periaguja o pericatéter; y el Kt de cada sesión, aportado por la función OCM del monitor Fresenius $4008 \mathrm{~S}^{\circledR}$.

Se realizaron analíticas cada 6 sesiones para la determinación del Kt/V (monocompartimental Daugirdas $2^{a}$ generación) y para la determinación del índice de recirculación. El dolor se cuantificó según una escala subjetiva de valores donde: $0=\sin$ dolor, $1=$ dolor leve, $2=$ dolor moderado y $3=$ dolor intenso ${ }^{3}$. El confort se valoró también mediante una escala subjetiva (0-4) en la que a mayor valor, mayor confortabilidad.

Se midió el tiempo de hemostasia basal y se registraron los tiempos de hemostasia en cada sesión. Se cualificó el estado de la cámara arterial y venosa mediante una escala numérica cuantitativa en la que: $0=$ cámara coagulada, 1=cámara con coágulo y 2=cámara limpia; y el estado del dializador, valorado igualmente con escala numérica, en la que $0=$ dializador coagulado, $1=$ dializador muy sucio, $2=$ dializador con muchos capilares coagulados, $3=$ dializador con pocos capilares coagulados y $4=$ dializador limpio.

\section{Análisis estadístico}

Se calcularon la media y la desviación estándar (DE) para cada valor y fueron analizados mediante el pro- grama informático SPSS 17.0 utilizando el análisis para datos pareados mediante test paramétricos y no paramétricos (t de Student y Prueba de Wilcoxon).

\section{Resultados}

Las características generales de los 10 pacientes estudiados han quedado recogidas en la tabla 1 . En la tabla 2 se han mostrado los resultados de la comparación entre la aguja convencional y el catéter fístula Supercath $A Z^{\circledR}$, de los parámetros dinámicos tales como PA, PV y PTM inicial y final. Un hallazgo relevante de estos datos es que la punción con el catéter Supercath $A Z^{\circledR}$ indujo a una disminución de la PA y PV estadísticamente significativo $(p<0,001)$ según muestra la figura 3 . El valor de la PTM también disminuyó aunque no alcanzó significación estadística.

En cuanto al estado de la cámara arterial, venosa y dializador finalizada la sesión, aunque sin significación estadística, sí mejoraron con el uso del Supercath $A Z^{\circledR}$, el estado de ambas cámaras fue de 2 (cámara limpia) en la escala numérica, mientras que en la aguja convencional fue de $1,98 \pm 0,6$ y 1,9 90,18 respectivamente, y el estado del dializador fue de $3,85 \pm 0,21$ en el Supercath $A Z^{\circledR}$ frente a $3,48 \pm 0,6$ ( $3=$ dializador con pocos capilares y $4=$ dializador limpio). Destacar que hemos observado una disminución de la dosis de heparina en pacientes puncionados con el catéter fístula $12,78 \pm 13,01$, respecto a la aguja convencional $15 \pm 15,02$, aunque sin significado estadístico.

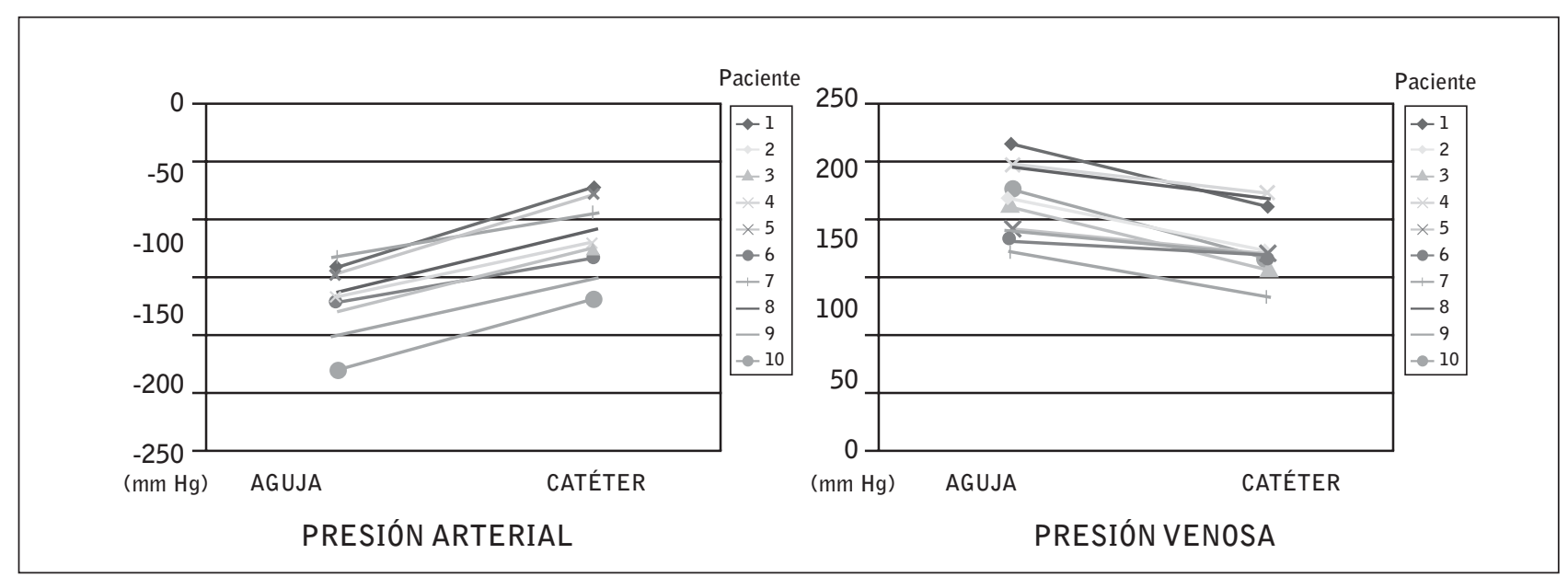

Figura 3. Presión Arterial y Presión Venosa medias finales 


\begin{tabular}{|c|c|}
\hline Sexo & V: $9(90 \%)$ M: $1(10 \%)$ \\
\hline Edad (Media \pm DE) & $69,3 \pm 10,36$ años ( $58-88$ años) \\
\hline $\begin{array}{l}\text { Tiempo en HD (meses) } \\
\text { (Media+DE) }\end{array}$ & $77,8 \pm 92,07$ ( $10-276$ meses) \\
\hline $\begin{array}{l}N^{0} \text { sesiones por semana } \\
\text { Tiempo medio }\end{array}$ & $\begin{array}{l}3 \\
232,5 \pm 12,74 \min (210-240 \mathrm{~min})\end{array}$ \\
\hline Causa de IRT & $\begin{array}{l}\text { No filiada: } 2(20 \%) \\
\text { Nefrosclerosis: } 2(20 \%) \\
\text { Nefropatía Diabética: } 2(20 \%) \\
\text { Glomerulonefritis: } 3(30 \%) \\
\text { HTA Maligna: } \quad 1(10 \%)\end{array}$ \\
\hline Comorbilidad & $\begin{array}{l}\text { HTA: } \quad 100 \% \\
\text { Cardiopatía isquémica: } 30 \% \\
\text { EVP: } \quad 30 \% \\
\text { HPT } 20: \quad 100 \% \\
\text { Trasplante previo: } 30 \%\end{array}$ \\
\hline Dializador & $\begin{array}{l}\text { FX 60: } 8(80 \%) \text { FX 80: } 2 \\
(20 \%)\end{array}$ \\
\hline Flujo Baño (ml/min) & 500: $8(80 \%) 800: 2(20 \%)$ \\
\hline Acceso vascular & $\begin{array}{l}\text { Prótesis: } 1(10 \%) \text { FAVI: } \\
9(90 \%) \text { : RC: } 3(30 \%) \text {, HC: } \\
6(60 \%)\end{array}$ \\
\hline Anticoagulación & $\begin{array}{l}\text { Heparina sódica: } 9(90 \%) \\
\text { HBPM: } 1(10 \%)\end{array}$ \\
\hline UF por sesión Aguja/catéter ${ }^{(1)}$ & 2.339,33 / 2.346,50 ml/sesión \\
\hline
\end{tabular}

Tabla 1. Características generales de los pacientes

\begin{tabular}{|l|c|c|c|}
\hline & AGUJA & SUPERCATH AZ & $\mathbf{p}$ \\
\hline PA inicial & $-130 \pm 25$ & $-82 \pm 27$ & $<\mathbf{0 , 0 0 1}$ \\
\hline PA final & $-142,7 \pm 24$ & $-98 \pm 25$ & $<\mathbf{0 , 0 0 1}$ \\
\hline PV inicial & $176,4 \pm 25$ & $144,5 \pm 21,5$ & $<\mathbf{0 , 0 0 1}$ \\
\hline PV final & $178,2 \pm 26$ & $146,8 \pm 24,6$ & $<\mathbf{0 , 0 0 1}$ \\
\hline PTM inicial & $43,3 \pm 9,2$ & $36,64 \pm 10,1$ & $\mathbf{0 , 0 2}$ \\
\hline PTM final & $42,6 \pm 17,3$ & $33,9 \pm 10,73$ & $\mathbf{0 , 0 1}$ \\
\hline
\end{tabular}

Tabla 2. Diferencias de los parámetros dinámicos PV, PA y PTM medias según cánula utilizada

El segundo hallazgo relevante que se ha encontrado es el aumento estadísticamente significativo del flujo sanguíneo real en la punción con Supercath $A Z^{\circledR},(p<0,01)$ según muestra la figura 4. Igualmente, con el catéter fístula, se ha observado un aumento de los litros dializados y el Kt por sesión y una disminución de la recirculación pero sin significado estadístico. Respecto al Kt/V no se ha registrado variación alguna. $Y$ por último mencionar la ausencia del colapso del flujo durante la diálisis con Supercath $A Z^{\circledR}$ frente a la existencia del mismo con la aguja convencional (tabla 3 ).

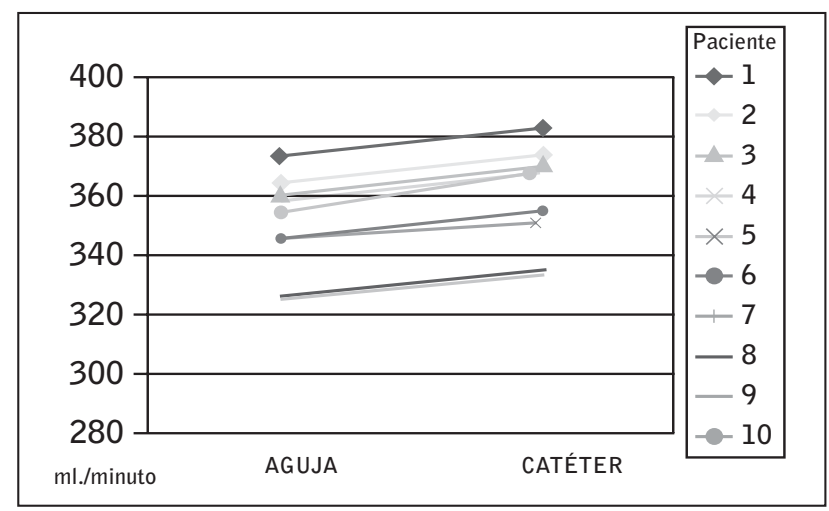

Figura 4. Flujo sangre real

\begin{tabular}{|l|c|c|c|}
\hline Flujo sangre bomba & AGUJA & SUPERCATH AZ & $\mathbf{p}$ \\
\hline Flujo sangre real & $384,5 \pm 21,4$ & $384,5 \pm 21,4$ & n.s. \\
\hline $\mathbf{N}^{0}$ litros dializados & $80,92 \pm 6,6$ & $83,22 \pm 7,34$ & 0,08 \\
\hline KT sesión & $47 \pm 3,86$ & $48,85 \pm 3,48$ & 0,03 \\
\hline KT/V medio & $1,64 \pm 0,29$ & $1,64 \pm 0,26$ & n.s. \\
\hline Recirculación & $7,13 \pm 7,86$ & $3,96 \pm 2,7$ & n.s. \\
\hline Colapso(1) & 7,63 & 0 & n.s. \\
\hline (1) $\mathbf{n}^{\text {0 de episodios por cada } 100 \text { sesiones de diálisis }}$ \\
\hline
\end{tabular}

Tabla 3. Comparación de la eficacia dialítica

En la tabla 4 se han recogido los datos en cuanto a las complicaciones por el uso de aguja convencional y Supercath $A Z^{\circledR}$. Hemos observado que sin diferencias significativas en las sesiones, con Supercath $A Z^{\circledR}$, los tiempos de hemostasia disminuyen, así como el resangrado postsesión. Destacar la ausencia de extravasación en el uso del catéter-fístula.

\begin{tabular}{l|c|c|c|} 
& AGUJA & SUPERCATH AZ & $\mathbf{p}$ \\
\hline $\begin{array}{l}\text { Resangrado post } \\
\text { sesión }\end{array}$ & 10,68 & 9,160 & n.s. \\
\hline Extravasaciones $^{(1)}$ & 0,76 & 0 & n.s. \\
\hline $\begin{array}{l}\text { Tiempo Hemostasia } \\
\text { Arterial }\end{array}$ & $4,76 \pm 1,87$ & $4,71 \pm 1,9$ & n.s. \\
\hline $\begin{array}{l}\text { Tiempo Hemostasia } \\
\text { Venosa }\end{array}$ & $4,89 \pm 2,29$ & $4,6 \pm 1,9$ & n.s. \\
\hline \begin{tabular}{l} 
(1) $^{\circ}$ de episodios por cada 100 sesiones de diálisis \\
\hline
\end{tabular}
\end{tabular}

Tabla 4. Diferencias de las complicaciones 
En la tolerabilidad, la aguja convencional ha mostrado un discreto mejor comportamiento 0,74 00,51 respecto al Supercath $A Z^{\circledR} 0,99 \pm 0,52$, aunque en ambos casos, la media de tolerancia fue muy buena, entre "sin dolor" (valor=0) y "dolor leve" (valor=1). En cuanto al confort durante la sesión ambas cánulas se aproximaron al máximo confort en la escala subjetiva de 0 a 4 , donde $3,81 \pm 0,36$ fue para la aguja convencional y 3,79 $\pm 0,23$ para el catéter fístula.

\section{Discusión}

Los resultados de nuestro estudio demuestran que en la diálisis el cambio de una aguja convencional por el catéter-fístula Supercath $A Z^{\circledR}$ permitió disminuir la PA y PV sin disminuir la eficacia dialítica, ya que el Supercath $A Z^{\circledR}$ presenta para la punción un calibre menor (16G) que la aguja convencional (15G) pero un mayor calibre interno (14G) lo que permite alcanzar mayores flujos reales.

Hemos encontrado en la bibliografía referencias a que es un hecho conocido que el aumento del calibre de la aguja permite mayor flujo de sangre $^{4-6}$ basándonos en esto, el uso del Supercath $A Z^{\circledR}$ permite optimizar el flujo de sangre real en cada paciente.

Si bien el pequeño tamaño muestral dificulta la consecución de resultados con significación estadística en algunos parámetros, sí resulta evidente la tendencia a mejorar en lo referente a complicaciones vasculares, lo que se justifica por la menor dosis de heparina necesaria y el menor trauma mecánico producido en el acceso.

La valoración del dolor en el paciente de HD ofrece aún bastantes dudas debido a que no existen instrumentos diseñados específicamente para detectar las necesidades de estos pacientes ${ }^{7}$, en nuestro estudio, se registra un aumento discreto del dolor con el uso del catéter fístula, la explicación que encontramos para ello ha sido la inexperiencia inicial en el manejo del Supercath $A Z^{\circledR}$ que indudablemente se ve reflejado con los datos.

Pacientes con accesos vasculares con gran flujo sanguíneo, que presentan habitualmente elevadas presiones venosas, pacientes con mayor tenden- cia a las complicaciones vasculares o problemas de hemostasia y pacientes que precisen técnicas dialíticas que pudieran mejorar con la utilización de flujos sanguíneos más elevados podrían ser los candidatos que más se beneficiarían con el uso de este catéter fístula.

Se necesitan nuevos trabajos con mayor tamaño muestral y mayor número de sesiones para evaluar adecuadamente el peso de estos hallazgos.

\section{Conclusiones}

1) Las diálisis con Supercath $A Z^{\circledR}$ muestran un mejor perfil hemodinámico que las realizadas con agujas convencionales.

2) El menor trauma mecánico en el acceso vascular con el catéter- fístula Supercath $A Z^{\circledR}$ permite disminuir la dosis de heparina sin empeoramiento en el control de la coagulación del dializador y del estado de las cámaras arterial y venosa.

3) El catéter Supercath $A Z^{\circledR}$ es tan eficaz como la aguja convencional en la adecuación dialítica y muestra una mejoría significativa en el número de litros dializados, número de episodios de colapso de flujo, y Kt, que en nuestro estudio no se ha visto reflejada en la mejoría del Kt/V

4) El catéter-fístula muestra una reducción de las complicaciones vasculares: extravasación y resangrado y un descenso del tiempo de hemostasia con respecto a la aguja convencional.

5) El uso del catéter-fístula es bien tolerado, aunque provoca un leve empeoramiento en la sensación dolorosa que acompaña a la punción. Su uso exige un período de aprendizaje por parte de la enfermería que la lleva a cabo.

6) La mejoría en los parámetros dinámicos abren las posibilidades de conseguir mayor eficacia dialítica al hacer factible la utilización de mayores flujos sanguíneos con presiones más adecuadas. 


\section{Bibliografía}

1. Herrero JA, García M. Accesos vasculares para hemodiálisis. En: Manual de hemodiálisis para personal de enfermería. ROCHE Edeka Med 2008: 69-81.

2. Hernández-López J. Accesos vasculares. Casos clínicos. En: Libro de comunicaciones del III Curso sobre Accesos vasculares para Hemodiálisis; Bilbao 24 y 25 octubre 2008.

3. Jarriz A, Bardales E, Pulido A, Albalate M. Efecto del calibre de la aguja sobre la eficacia del la Hemodiálisis. Rev Soc Esp Enferm Nefrol 2006; 9 (2): 128-131.

4. Gallego E et al. Efecto del calibre de las agujas sobre la recirculación y la eficacia de la HD. Nefrología $1997 ; 16: 322$.
5. Sánchez Villar I et al. Calibre de la aguja y calidad de la hemodiálisis. Comunicación en el XXIV Congreso de la SEDEN. Valencia. 1999; 120-124.

6. Tovar A et al. Efecto del cambio de aguja sobre la eficacia de la hemodiálisis, las presiones del circuito y la recirculación. Comunicación en el XXI Congreso de la SEDEN. Salamanca. 1996; 204- 210.

7. Rodriguez MA, Hernández $D$, Gutiérrez $M J$, Juan $F$, Calls J. Evaluación del dolor crónico en una Población de pacientes hemodializados. Rev Soc Esp Enferm Nefrol 2007; 10 (2): 137-143. 